\title{
Brightness of paired photic pulses with pulse duration and separation varied'
}

FREDERICK C. BLECK, LEIIGH UNIVERSITY

EUGENE A. CRAIG, ${ }^{2}$ CALIFORNIA STATE COLLEGE

The brightness of pairs of photic pulses, differing in pulse duration and pulse separation, was measured. The dato were in general agreement with the neurophysiological model of Bishop and Clare. Pulse duration appeared to have little systematic influence on the shape of the curves. Time between onset of the first and second pulse appeared to be a prime factor determining the receptivity of the cortex to the second pulse. The two pulses were seen as single below about $.03 \mathrm{sec}$ separation. The second pulse was always judged brighter than the first.

The present report is concerned with an experimental test of the ability of a neurophysiological model to predict perceptual phenomena. The experiment involved obtaining measures of the brightness of pairs of photic pulses under different conditions of pulse duration and pulse separation. The procedure for reporting will be to present a brief summary of the model; to develop predictions from the model, and then to report the experiment.

\section{THE MODEL}

\section{Summary of the Model}

It has been noted that an isolated shock to the optic nerve of the rabbit is followed by an extended sequence of changes in the evoked potential of the optic cortex in the form of a train of waves of gradually decreasing amplitude. A resemblance can be noted between the latter period of this evoked potential and the human cortical "alpha rhythm" (Bishop, 1936). Brazier (1962), recording human EEGs with the aid of a computer, has presented data showing long lasting wave trains following an isolated flash to the retina (Fig. 1). Bishop and Clare have constructed a model of cortical processes consistent with such findings (see Bartley, 1959). The model has relevance not only for single pulse phenomena but for paired pulses as well.

Briefly, the model operates as follows: energy impinging on the retina traverses the optic nerve and tract and synapses on the lateral geniculate body. From the geniculates the impulses travel to the fourth layer of the occipital cortex where they synapse on the short axon Golgi cells. The Golgi cells in turn synapse on large pyramid cells of the same layer.

From the pyramid cell the impulse follows two paths. One path is down the pyramidal axon to the thalamic cells. The second path

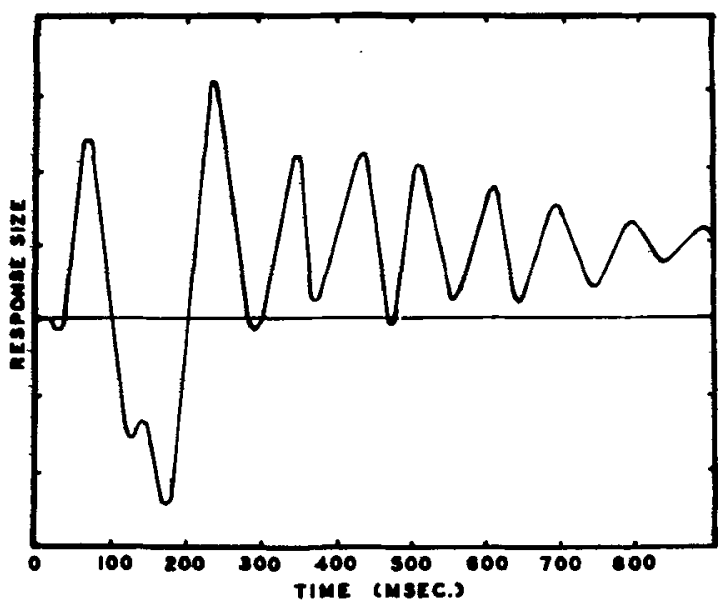

Fig. I. Long lasting train of waves following a single flash (from Brazier, 1962). involves recurrent branches of the pyramid cell. These branches arborize in the cortex and synapse on a second set of Golgi cells. The second path terminates by activating the dendrite of the pyramid cell and passing down the axon to the thalamus. The time of the first path to the thalamus is of the order of $20 \mathrm{msec}$. The second path is longer and it is thought that, from the first Golgi cell to the thalamus, the interval is equivalent to the alpha cycle of the specie involved.

If an incoming impulse along the optic nerve arrives at the thalamus at the same instant that an impulse is returning to the thalamus along corticofugal loops, the result is a facilitation of the thalamic celis upon which these branches terminate. If the optic nerve activity reaches the thalamus at any other time there will be no facilitation and consequently lower cortical potential.

Other investigators have offered data on the neurophysiological effects of paired photic pulses that are consistent with the Bishop and Clare model. Bartley (1941) has shown that the amplitude of the evoked potential of a second pulse waxes and wanes as a function of the separation between pulses. He found that, in order to obtain an amplitude at the cortex for the second pulse as great as that of the first pulse, the pulses had to be separated by an interval equivalent to the organism's alpha cycle. Bremer (1961) has reported similar findings.

\section{Predictions from the Model}

According to the model, the cortical potential reflects not only the effects of the photic pulse but the state of cortical and corticofugal activity as well. If, then, an afferent impulse reaches the thalamus at the same time as descending corticofugal activity, the resulting potential changes arriving at the first Golgi cell should be facilitated. In like manner, a pulse reaching the thalamus at a time between descending corticofugal bursts will not be facilitated and the resulting cortical evoked potential will not be as great. If we make the assumption that the amplitude of the cortical evoked potential determines the quantity, or intensity, of the perception of a given stimulus and we accept Brazier's data as the generalized case, then, the expectation is that sensory brightness curves for paired photic pulses should run parallel with her curve (Fig. 1). Allowing for time of descent to the thalamus, the brightness curves should wax and wane in like manner but be out of phase with her curve.

Concerning the relative brightness of a pair of pulses, the neurophysiological model suggests that the second pulse should be perceived as brighter than the first only when the second coincides with the maximum corticofugal activity. Again using Brazier's curve as the example, the second pulse should appear brighter than the first between 50-100 msec and between 100-280 msec. In all other cases the first pulse should appear brighter.

A third prediction deals with the point at which the two pulses can no longer be perceived as separate. Bartley implies that this critical point for man lies around $.1 \mathrm{sec}$ separation between pulses. Boynton (1961), however, has shown that differences in sensory threshold are obtainable for pulses separated by as little as $.03 \mathrm{sec}$. Bartiey's own data from the rabbit shows pulses as close together as $.08 \mathrm{sec}$ in the record. This value is considerably lower than the $.2 \mathrm{sec}$ alpha cycle for that specie. Generalizing to the human, separations of pulses by as little as $.04 \mathrm{sec}$ should be detectable. Bartley (1961) has suggested that the signal for the cessation of a pulse is the "off" response. If pulses are presented with an interval less than $.03 \mathrm{sec}$ the "off" response is inhibited and fusion is perceived. On the basis of these data, one would expect that the critical separation at which the distinction between two pulses can no longer be seen would lie near $.03-.04 \mathrm{sec}$. 
The above discussion and predictions are based on Bartley's 1941 presentation. Later statements by Bishop (1949) and others (Brazier, 1958; Morison \& Dempsey, 1943) have taken the position that it is not necessary to infer reverberatory closed circuits to account for the alpha excitability cycle. Instead these authors stress that the cycle is due wholly to intracortical mechanisms. The predictions tested in the present experiment, however, remain relevant with either explanation for such excitability cycles.

An additional expectation, not based on the model, is that the degree of brightness enhancement for paired pulses should vary as a function of pulse duration. This prediction follows from the fact that maximum brightness for a single photic pulse of moderate intensity (Broca-Sulzer phenomenon) occurs with a pulse duration of about .05 sec. Such enhancement should also be apparent for two similar pulses occurring in close temporal proximity.

\section{METHOD}

\section{Apparatus}

The apparatus, which employed an episcotister to interrupt a tungsten light source, was so designed as to produce two adjacent 1 deg targets separated by $5 \mathrm{deg}$ in an adapting field. The left target consisted of two photic pulses appearing consecutively at the same locus. The pulses were variable by the $E$ with respect to duration and interval between pulses. The right target was a continuous source. The $S$ could vary it in luminous intensity by means of neutral density filters over a range from $.06 \mathrm{ft}-\mathrm{c}$ to 60 $\mathrm{ft}-\mathrm{c}$.

\section{Subjects}

Two Ss were tested; one male (age 27) and one female (age 24). Both had 20/20 visual acuity with or without glasses.

\section{Procedure}

The basic task of the Ss in this experiment was to match the brightness of a pair of pulses, with pulse duration and interval between pulses varied, with a similas steady target that was variable in terms of luminous intensity. Initially it had been planned to make the matches in reference to the second pulse alone. Because of the extreme difficulty of making such judgments reliably, it was decided to modify the procedure by making the matches on the basis of both pulses taken together. It was assumed that the effect of the initial pulse would be constant and that any changes in brightness could be attributed solely to the second pulse.

Pulse separation was varied in $.02 \mathrm{sec}$ steps from .02 through $.24 \mathrm{sec}$. Four pulse durations were employed: $.025, .050, .075$, and .100 sec. All combinations of pulse separation and pulse duration were tested for both Ss with the exception of the .100 sec pulse duration for which data was obtained on only one $S$. The psychophysical method of adjustment was used with five ascending and five descending trials for each pulse separation. Each pair of pulses was separated by $1 \mathrm{sec}$ before the two pulses were again presented. A given series of pulses was continuously presented until the $S$ matched their brightness with that of the steady source. As supplementary information, the extent of the BrocaSulzer phenomenon was measured for the Ss by having them make brightness matches between single pulses differing in duration and the continuous source.

\section{RESULTS}

The results are shown in Figs. 2 through 5. Brightness taken strictly as a function of pulse separation (Fig. 2) did not yeild a systematic set of data. The only apparent consistencies were a reduction in variability as pulse duration increased and the observation that, if any systematic excursion above or below one standard deviation unit from the base line is taken as a positive or negative peak, then with the exception of the $.100 \mathrm{sec}$ pulse duration, the curves for both Ss under the various pulse durations generally show two positive and two negative peaks.

Brightness was also plotted as a function of time between the onset of the first pulse and the onset of the second puise. These
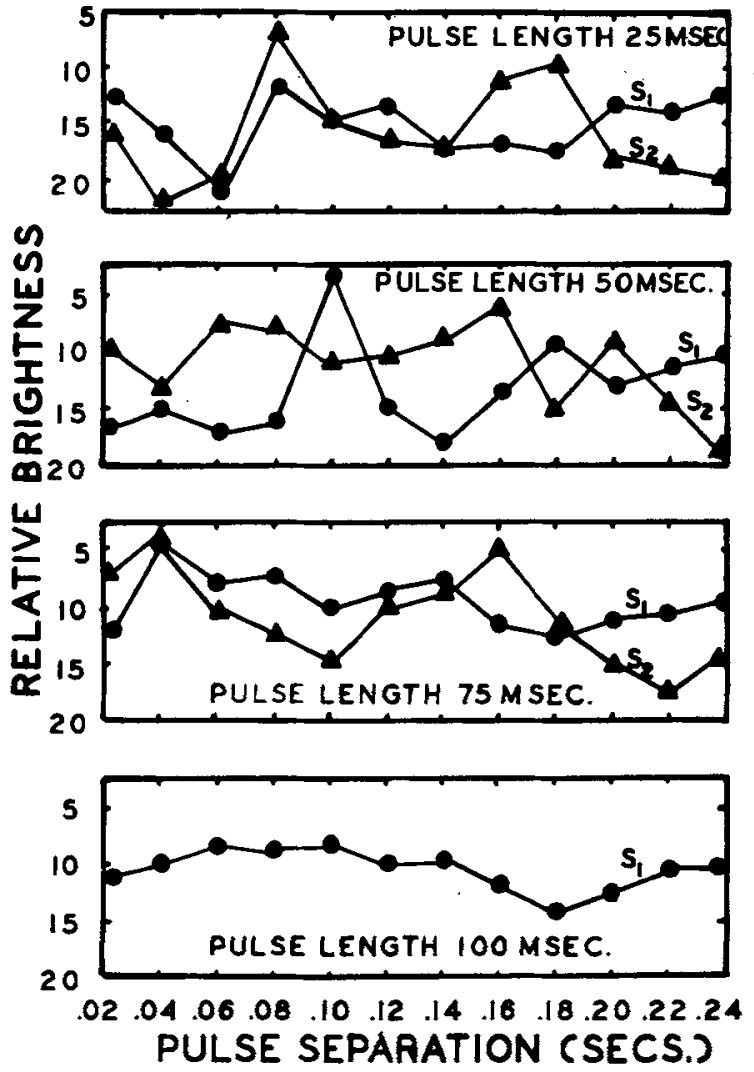

Fig. 2. Relative brightness as a function of pulse separntion.

data (Fig. 3) suggest that puise onset is the prime factor in determining the receptivity of the cortex to the second pulse. It can be seen that: (a) below 60 msec between onsets, brightness values fall near the group mean, (b) between $60-100 \mathrm{msec}$ they are typically below the mean, (c) between $100-150$. msec they are above the mean, (d) between $150-200 \mathrm{msec}$ they are typically at the mean, (e) between $200-250 \mathrm{msec}$ they are again above the mean, and (f) beyond $250 \mathrm{msec}$ they faill at or below the group mean.

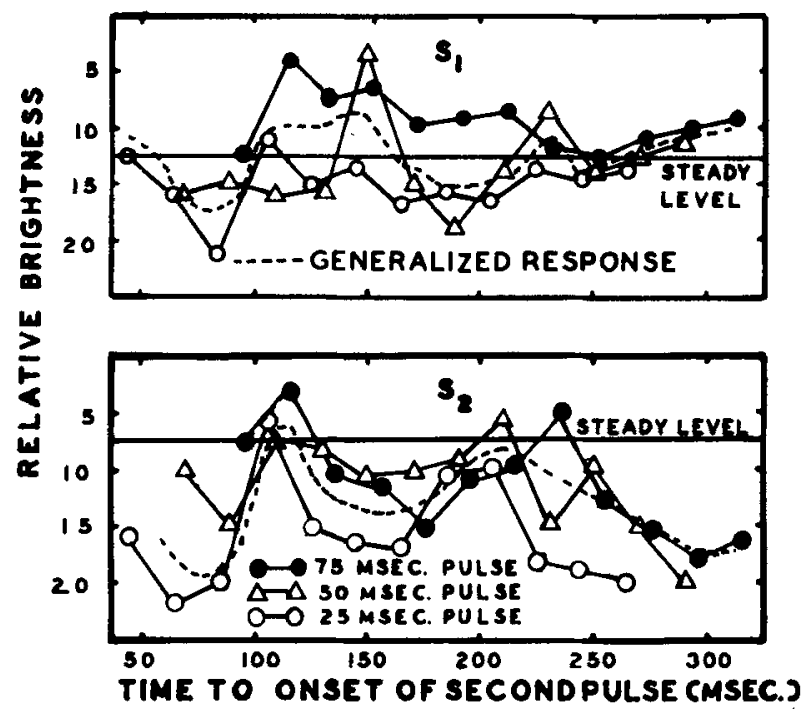

Fig. 3. Relative brightness as a function of time between onset of the first pulse and onset of the second pulse. 


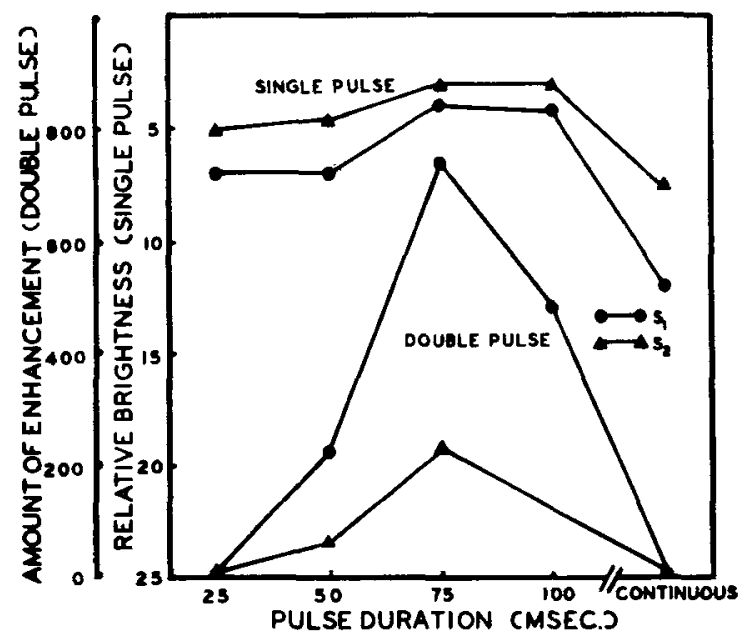

Fin. 4. Rebative brightneas of single pulnes, and amount of enhancement of paired pubes, for different pulse durations.

The time between positive peaks as well as the average time between depressions is about $100 \mathrm{msec}$. The interval from onset of the first pulse to the first peak is slightly greater than $100 \mathrm{msec}$ in all cases.

The two pulses were always perceived as one for the $.02 \mathrm{sec}$ pulse separation condition and always perceived as two for the .04 sec condition. In order to identify the transition zone more clearly, an intermediate value of $.03 \mathrm{sec}$ separation was also tested. This separation was perceived by $S_{1}$ in $67 \%$ of the trials and by $S_{2}$ in $61 \%$ of the trials.

The second pulse was always reported as brighter than the first by both Ss under all pulse separations and pulse durations.

Brightness matches were also made between the continuous source and single pulses of different durations. Figure 4 shows this data in the upper set of curves. It can be seen that a typical Broca-Sulzer function was plotted with maximum brightness occurring for pulse lengths between 75 and $100 \mathrm{msec}$. The lower curves of that figure represent the amount of brightness enhancement as a function of pulse duration for paired pulses. The points were computed by summing the area of each curve above the match of the constant intensity test target. The curves are only generally similar but indicate a brightness peak at 75 msec.

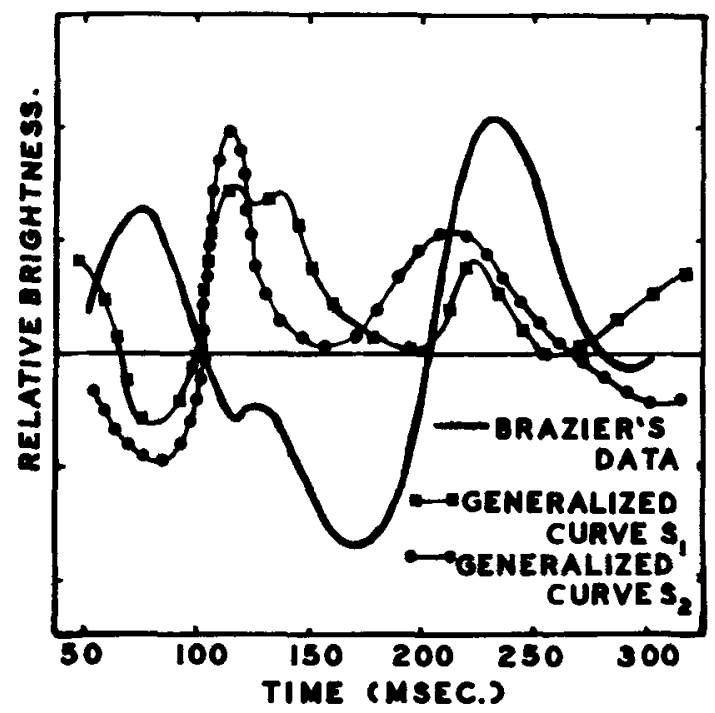

Fig. 5. Generalized brightness curves from data of the present experiment compared with Brazier's data.

\section{DISCUSSION}

The data agree with the model of Bishop and Clare in the following ways:

(a) There is a brief initial increase in brightness for short pulses separated by intervals of less than 50 msec. This early facilitation could be due to the initial impulse traveling through the first Golgi and pyramid cells then directly down the corticofugal fibers to the thalamus.

(b) A second facilitatory peak was predicted due to the portion of the impulse traveling along the recurrent branches of the pyramid cells and into the upper layers of the cortex. The duration between pulse onsets necessary to take advantage of this corticofugal activity is just greater than one alpha cycle. This second enhancement peak was found to occur slightly beyond the alpha rhythm cycle for both $S_{s}\left(.125 \mathrm{sec}\right.$ for $S_{1}$ and $.112 \mathrm{sec}$ for $\left.S_{2}\right)$.

(c) According to the model, this second corticofugal wave is strong enough to recirculate and cause a third wave capable of facilitating incoming afferent activity. In this case the predicted duration would be slightly greater than two alpha cycles in order for the second pulse to take advantage of the facilitation. This is also supported by the data. The enhancement peak occurred at $.230 \sec$ for $S_{1}$ and $.225 \sec$ for $S_{2}$.

Figure 5 represents the generalized brightness curves from this experiment in comparison with Brazier's single-flash data. The curves are somewhat similar in terms of peaks and depressions but are not in phase with each other. The reason for the differences is not apparent but may be due to differences in method or in stimu lus parameters such as target size, pulse duration, or intensity level.

The fact that sensory brightness curves can be best specified in terms of the difference in time between the onset of the first pulse and the onset of the second pulse, under all pulse durations, indicates that pulse duration plays little part in determining the location of the peaks and depressions of the curves. The initial portion of the pulse appears to be primarily effective with the latter portion contributing little to the cyclical response of the cortex. This does not mean, however, that pulse duration is without effect. The similarity between the Broca-Sulzer curves for single pulses and the enhancement curves for double pulses (Fig. 4) indicates that the level of a particular peak or depression is a function of pulse duration. The data suggest that two factors are acting to produce brightness level: the cortical excitability cycle of Bishop and Clare, and the afferent discharge density occurring during these positive cycles. Granit (1955) has shown that the Broca-Sulzer phenomenon can be explained in terms of discharge density.

The finding that two pulses cease to be seen as two for separations below $.03 \mathrm{sec}$ agrees with the prediction made on the basis of the neurophysiological data and further suggests that the "off" response is responsible for detecting differences between pulses. Bartley, Granit, and others have indicated that the "off" responses are inhibited below about $.04 \mathrm{sec}$. This finding is not in agreement, however, with Bartley's suggestion that, for periods much less than the alpha cycle, the incoming afferent pulses cannot reach the cortex. It does, though, suggest that brightness is a function of some periodic activity similar in nature to the alpha rhythm. Even though the apparent brightness of the target is reduced under certain temporal relations, information may still be transmitted during these periods.

The repeated designation of the second of the paired pulses as the brighter of the two, independent of pulse duration, does not agree with the model. The reason for this discrepancy is unknown and requires additional investigation.

In conclusion, then, the data generally support the assumption that brightness is related to the amplitude of the cortical evoked potential and that the model of Bishop and Clare reflects a fair approximation of the state of affairs in the occipital cortex.

\section{REFERENCES}

BARTLEY, S. H. Vision: $A$ study of its basis. New York: Van Nostrand, 1941. 
BARTLEY, S. H. Central mechanisms in vision. In J. Field, H. W. Magoun, \& V. G. Hall (Eds.), Handbook of physiology, Sec. 1, Neurophysiology, Vol. 1. Washington, D. C.: Amer. Physiol. Soc., 1959. Chapter 30, pp. 713-741.

BARTLEY, S. H., NELSON, T. M., \& RANNEY, J. The sensory parallel of the reorganization period in the cortical response to intermittent retinal stimulation. J. Psychol., 1961, 52, 137-147.

BISHOP, G. H. The interpretation of cortical potentials. Cold Spr. Harb. Symp. Quant. Biol, 1936, 4, 305-318.

BISHOP, G. H. Phenomena in thalamus and cortex. EEG Clin. Neurophysiol. $1949,1,421436$.

BOYNTON, R. M. Some temporal factors in vision. In W. Rosenblith (Ed.), Sensory communication. Cambridge: M.I.T. Press, 1961. Pp. 739-756.

BRAZIER, M. A. B. Studies of evoked responses by flash in man and cat. In H. Jasper, L. Procter, R. Knighton, W. Noshay, \& R. Castello (Eds.), Reticular formation of the brain. Boston: Little, Brown \& Co., 1958.

BRAZIER, M. A. B. The analysis of brain waves. Scient. American, June, $1962,2-10$
BREMER, F. Neurogenic factors influencing the evoked potential of the cerebral cortex. In W. Rosenblith (Ed.), Sensory communication Cambridge: M.I.T. Press, 1961. Pp. 675-698.

GRANIT, R. Receptors and sensory perception. New Haven: Yale University Press, 1955.

MORISON, R. S., \& DEMPSEY, E. W. Mechanisms of thalamocortical augmentation and repetition. Amer. J. PhysioL, 1943, 138, 297-308.

\section{NOTES}

1. This study was supported by Grant MH 07179 from the National Institute of Mental Health, U.S. Public Health Service.

2. Address: Department of Psychology, California State College, California, Pa. 15419.

(Accepted for publication June 24, 1968.) 